Revue

Revue de l'histoire des religions

de Ihistoire des religions

\title{
La formation de la « nation » juive dans l'Europe chrétienne latine
}

Des « restes d'Israël » à la « communauté sainte »

The Formation of the Jewish "Nation" in Latin Christian Europe: from the

"Remnants of Israel" to the "Holy Community"

Sylvie Anne Goldberg

(2) OpenEdition

Journals

Édition électronique

URL : http://journals.openedition.org/rhr/8736

DOI : ERREUR PDO dans /localdata/www-bin/Core/Core/Db/Db.class.php L.34 : SQLSTATE[HY000]

[2006] MySQL server has gone away

ISSN : 2105-2573

Éditeur

Armand Colin

Édition imprimée

Date de publication : 1 juin 2017

Pagination : 237-254

ISBN : 978-2-200-93126-1

ISSN : 0035-1423

Référence électronique

Sylvie Anne Goldberg, « La formation de la « nation » juive dans l'Europe chrétienne latine », Revue de I'histoire des religions [En ligne], 2 | 2017, mis en ligne le 01 juin 2019, consulté le 08 janvier 2021. URL http://journals.openedition.org/rhr/8736 ; DOI : https://doi.org/10.4000/rhr.8736 


\title{
La formation de la « nation » juive dans l'Europe chrétienne latine Des « restes d'Israël » à la « communauté sainte »
}

\begin{abstract}
Cet article vise à éclairer les usages dont les notions de " restes d'Israël », de « communauté sainte » et de "nation juive » ont fait l'objet au cours des temps : notions exégétiques pour les deux premières, phénomène historique pour la troisième. L'analyse cherche à associer des éléments qui relèvent de genres distincts : la théologie (anhistorique) et l'histoire (ancrée dans le temps) afin de mettre en lumière le glissement sémantique qui, dans la constitution historiographique du fait juif dans l'Europe chrétienne et dans la production d'une définition des groupes juifs, a conduit aux conceptions modernes des notions de "communauté » et de "nation». Mis en relation avec l'idée de rédemption collective et de sainteté, ce glissement permet de saisir l'importance accordée par la pensée chrétienne à la conservation des vestiges d'Israël en vue de leur conversion ultime.
\end{abstract}

\section{The Formation of the Jewish "Nation" in Latin Christian Europe: from the "Remnants of Israel" to the "Holy Community"}

The reflection presented in this article aims at clarifying the uses that the concepts of "remnants of Israel" she'erit Yisrael), "holy community" (qehillah qedushah) and "Jewish nation" have been made of over time. The first two are exegetical notions; the third notion refers to a historical phenomenon. The analysis therefore seeks to combine elements belonging to two different realms : theology (ahistorical) and history (anchored in time), in order to highlight the semantic shift that occurred in the historiography of the constitution of the Jewish fact in so-called Christian Europe and in the production of a definition of Jewish groups. This shift, which led to the modern conceptions of the notions of " community " and "nation », is related to the issue of collective redemption (the "remnants") and holiness ("qedushah") and thus captures the importance that Christian thought accords to the conservation of the remains of ancient Israel for their ultimate conversion. 
Assure, ô Seigneur, le salut de ton peuple, des derniers restes d'Israël (hallelu vé-imru hocha [...] et-amekha et-ché'érit yisrael).

Je les ramènerai du pays du Nord et les rassemblerai des confins de la terre [Jérémie, 31, 7-8].

En ce jour $[\ldots]$ je recueillerai, je rassemblerai tout Jacob, je mettrai ensemble les restes d'Israël, (kulekha qabets eqabets che'erit yisrael) [Michée, 2, 12].

On a souvent tendance à oublier que les mots et les concepts que l'on emploie usuellement sont parfois héritiers de notions qui n'ont plus cours, et ont été transformés par les siècles. Il en va ainsi des termes «communauté » et "nation » qui, si elles renvoient à des constructions sociales et politiques contemporaines, portent en elles des significations léguées par l'histoire. Avant d'intégrer les catégories juridiques définissant ce qu'est ou doit être un corps social dans le monde moderne ou la Cité, les principes régissant les assignations ethniques, l'adhésion à un mode de vie religieux ou l'obtention d'une citoyenneté (géographique ou politique) signalaient les différentes couches de population évoluant dans les mondes pluriels des sociétés antiques. Habitants d'une région, adeptes d'une religion, ou bénéficiaires de droits particuliers, ces groupes sociaux ont fini par répondre à des critères de plus en plus précis qui, à leur tour, ont conduit à la détermination de définitions toujours plus fixes. Un cas exemplaire de ces glissements est fourni par le thème de ce dossier sur les Juifs et la nation. Si on considère le mot «nation », on constate ainsi que les habitants de la province de Judée, appelés ioudaioi se sont mués au cours du temps en juifs, tout en gardant la même désignation de ioudaioi, définissant alors plus largement tant une origine régionale et/ou ethnique que religieuse ${ }^{1}$. Il en va de même de la notion de «communauté » qui, dans sa lente construction médiévale, est venue se superposer à ce qui, jadis, était plutôt entendu comme une «association », une

1. Annette Yoshiko Reed, «Ioudaios before and after Religion», Timothy Michael Lax and Charles Halton (éds), Jew and Judean: A Forum on Politics and Historiography in the Translation of Ancient Texts, Marginalia Review of books, August 2014, p. 21-26. 
« colonie » voire une «implantation». Actuellement, à entendre les médias, ce serait la référence à une affiliation identitaire édifiée sur une distinction religieuse qui primerait dans l'idée que l'on se fait d'une «communauté ». En analysant la manière dont les termes «communauté » et «nation » se sont appliqués aux Juifs, à partir des acceptions que ces termes revêtaient dans différents contextes historiques, cet article tente une réflexion sur le sens que découvrent ces mutations sémantiques.

La réflexion que je vais présenter dans cet article s'articule autour de trois termes: chéérit Yisrael, qehillah qedouchah, et nation. Elle vise à éclairer les ressorts des différents usages dont ils font l'objet au cours des temps. Il existe, on le sait, un quasi-consensus historique sur le fait que l'institution communautaire juive (en tant que représentation formelle du groupe local) émerge en Europe occidentale à partir du $\mathrm{X}^{\mathrm{e}}$ siècle. Sur l'origine et la manière dont ce modèle - qui s'amplifie tout au long du Moyen Âge pour connaître une forme d'apogée à partir de la période pré-moderne - s'est finalement imposé, le consensus est moins certain. Pour simplifier la question, on pourrait dire que deux contenus sémantiques distincts coexistent dans l'idée de «nation». Ainsi, dans la langue hébraïque, les notions de « restes d'Israël » (ché'érit Yisrael) et de « communauté sainte » (qehillah qedouchah) reflètent des notions exégétiques. Celle de «nation juive» renvoie, pour sa part, à un phénomène historique. Les deux premières notions sont liées à des évolutions internes au judaïsme, la seconde est externe.

Ma réflexion cherche donc à associer deux éléments qui n'ont a priori pas grand-chose en commun et relèvent de catégories distinctes : la théologie (anhistorique) et l'histoire (ancrée dans le temps) afin de mettre en lumière le glissement sémantique qui s'est produit et a conduit aux conceptions modernes des notions de « communauté » et de «nation ». Théologie et histoire s'allient en effet dans la constitution historiographique du fait juif dans l'Europe dite chrétienne et dans la production d'une définition des groupes juifs en «nation juive ». Et il en va de même pour l'idée de «communauté » juive, institution reconnue et organisée depuis une date indéterminée au cours du $\mathrm{X}^{\mathrm{e}}$ siècle jusqu'à son abolition dans les processus d'émancipation. 


\section{LE LEGS DE L'EXÉGÈSE}

Commençons par les notions exégétiques. Celle de «restes d'Israël» (che'erit/ che'arah) s'énonce dans la littérature prophétique, "écoute maison de Jacob et restes d'Israël » (Isaïe $46,3)^{2}$. Elle s'interprète comme l'ensemble du peuple «qui reste » après sa déportation ou revient sur sa terre, dans la perspective d'Isaïe, et son che'ar yachuv. Cette notion de destin «collectif» du peuple d'Israël se développe également avec l'idée d'un «tout» ou d'un «ensemble», qehal, que l'on trouve sous la forme de qehallah: "Achour et l'ensemble de son peuple» dans Ézéchiel 32, 22; ou de qehillah: «Je convoquais une grande réunion/ assemblée », dans Néhémie 5, 7. Le Targum Palestinien traduit ainsi le verset d'Isaïe: "En ce temps-là, les restes d'Israël et les rescapés de la maison de Jacob ne s'inclineront plus devant ceux qu'ils servent»; et il précise dans les deux versets suivants que ces «restes» et «rescapés» sont ceux qui ont évité de pécher ${ }^{3}$. Ce transfert de la notion des «restes» d'Israël à celle de la communauté contient un sens qu'il est aisé d'appréhender à partir des sources talmudiques. Shmuel Safrai a ainsi relevé dans le Talmud Babylonien six occurrences renvoyant à la qehillah qedouchah de-vei Yeruchalayim, la communauté sainte de Jérusalem, qui aurait existé à la fin du II $^{\mathrm{e}}$ siècle. La question insoluble de savoir si ce Saint ensemble regroupait des sages, des piétistes de l'époque, voire des réfugiés de Jérusalem détruite et interdite est hors de ce propos. En revanche, il est important de relever que, selon la tradition transmise par les géonim, cet ensemble serait celui que l'on retrouve également sous l'appellation de 'eda qedouchah dont deux membres sont identifiés dans le Ma'asser cheni du Talmud Palestinien, comme le rapporte Rachi dans un commentaire ${ }^{4}$ : "J'ai trouvé dans les responsa de notre rabbi Cherira de mémoire bénie, la définition de ces Sages que l'on appelait la Communauté Sainte de Jérusalem [...], au nom de la Sainte Communauté de Jérusalem, rabbi Simeon ben Eliakim affirmait que celui qui combinait rédemption et prière ne

2. Également Jérémie, Ézéchiel 11,13, Michée, Aggée 1, 12-14, Sophonie 2, 3-7, Esdras 9, 8, 14-15.

3. Targ. Yonathan, 10, 20-22.

4. Diqdukei soferim sur Betsa, p. 68. 
serait pas en danger. Rav Yehudaï Gaon, de mémoire bénie, dit, en citant des rabbins précédents, que rabbi Siméon ben Menasia et rabbi Yosé ben Ha-Mechulam consacraient le tiers de leur journée à la Torah, un tiers à la prière, et un tiers au travail, et que ces hommes formaient la Sainte Communauté de Jérusalem ${ }^{5}$ ». La même anecdote est rapportée dans $Q o R$ où Rabbi dit, à propos de la Sainte Communauté ('eda qedouchah) : «Pourquoi les appellet-on Sainte communauté ? Parce que là-bas, il y avait rabbi Yosé ben Mechulam et rabbi Simeon ben Menasia qui divisaient leur journée, en un tiers en Torah, un tiers en prières, et un tiers en travail...6 ${ }^{6}$. La participation au "groupe »-quel qu'il ait pu être - repose donc sur une certaine idée de la sainteté. Celle-ci se précise à la faveur des distinctions apportées au nom de Rabbi Yehouda dans le midrach Siffrei «Il existe quatre [formes de] qahal [groupes ou communautés], le qahal des prêtres, le qahal des Levi, le qahal d'Israël, le qahal des convertis et des Sages ${ }^{7} »$.

Le rôle d'Eusèbe Pamphile dit de Césarée dans la constitution de l'identité chrétienne n'est plus à démontrer. Indissociable de l'identité du christianisme, la représentation des Juifs et du judaïsme y exerça une fonction importante, à la transformation de laquelle Eusèbe participa grandement. Afin d'assurer les bases théologiques du concept du «vrai Israël » devant supplanter l'Israël déchu, Eusèbe s'est penché sur la notion des « restes ». Se fondant sur les versets d'Isaïe $(10,20-22)$ : «En ce jour le reste d'Israël (che'ar yisrael) et les débris de la maison de Jacob [...] Un reste, un reste de Jacob (che'ar yachuv che'ar Yaakov) retournera vers le Dieu Puissant [...] il n'y aura plus qu'un reste pour s'amender »; Eusèbe, reprenant les prémisses posées par Origène ${ }^{8}$, distingue dans la Démonstration évangélique «l'ensemble»du peuple juif, rejeté par Dieu, de son « reste », qu'il définit comme étant les élus qui ont accepté la prédication évangélique9. Ce

5. Shmuel Safrai, "The Holy Congregation in Jerusalem", Scripta Hierosolymitana, vol. 23, 1972, p. 62-74, 67.

6. Qohelet Rabba 9, 9. La confirmation du Rashi, Otzar ha-geonim, I, Berakhot, 23.

7. Siffrei Devarim, Ki tetseh, pisqa remez, p. 4-5.

8. Sébatien Morlet, «Le reste d'Israël » selon Eusèbe de Césarée : théologie, exégèse et histoire d'une catégorie frontalière », Simon Mimouni, La croisée des chemins revisitée, Paris, Cerf, 2012, p. 275-297.

9. Démonstration Évangélique, II, 3, $78 ; 108 ; 161$. 
« reste » est, selon lui, composé des apôtres, des disciples et des évangélistes qui ont servi de courroie de transmission entre la révélation originelle faite à Israël, son rejet actuel et historique, et la continuité entre l'ancien et le nouvel Israël incarnée par l'émergence du christianisme. Ce renversement de l'interprétation de la promesse messianique contenue dans la prophétie d'Isaïe sépare le «tout » du « reste », en renvoyant l'ensemble du peuple juif dans une zone de relégation, dont seul, le « reste » sera sauvé.

Tout, ou partie d'un tout, la «communauté » peut, dans les textes juifs, renvoyer à un ensemble ou un sous-ensemble allant du plus large au plus restrictif : elle rassemble ceux qui ont échappé à la disparition, ceux qui n'ont pas succombé au péché, ou encore ceux qui s'associent dans certains exercices de piété. À Athènes, l'Ekklesia était la maison du peuple. Symbole de la démocratie athénienne, elle était ouverte à tous les mâles. C'est par ekklesia que les auteurs des Septante ont choisi de traduire le qahal de la Bible hébraïque (parfois utilisant sunagôgê «maison de l'Assemblée » au sens profane : beit ha-knesset), et c'est ce même terme, simplement transposé en latin qui indiquera, plus tard, tout lieu de culte, aussi bien juif que chrétien, avant de se réduire à désigner l’Église.

\section{LES LEÇONS DE L'HISTOIRE}

Passons maintenant aux notions extérieures. Du point de vue de l'histoire, la catégorie collective des Iudaei (« Juifs ») est une construction juridique qui apparaît à la fin de l'empire romain, parmi les lois compilées dans le Code de Théodose (438-439). Alors qu'ils avaient jusque-là bénéficié de la reconnaissance légale de l'exercice de leur culte ethno-national en tant que gens, natio, ou secta, les Juifs, pour ceux qui n'en bénéficiaient pas encore, obtinrent la citoyenneté romaine, comme tous les autres pérégrins de l'Empire par la Constitution antonine de 212. Cette catégorie de la population se forme par le processus de mutation de l'ancien droit romain païen en droit romain chrétien. Ce processus se déroula jusqu'au $\mathrm{VI}^{\mathrm{e}}$ siècle avec la promulgation du Code Justinien, qui prit effet, en Orient, le 16 avril 529, en abrogeant le Code théodosien, entré en vigueur (en Orient) le 
15 février 438. Entre ces deux corpus juridiques dont les influences sont différentes, le Bréviaire, imposé en 506 par les Visigoths, ouvre à des distinctions juridiques au sein de la chrétienté ${ }^{10}$. En simplifiant à l'extrême, on peut dire que l'approche à l'égard des Juifs est prise entre deux conceptions contradictoires : l'une, illustrée par les positions de l'empereur Théodose en 390 et en 393, est héritière immédiate de la jurisprudence romaine et les protège contre toute agression à l'instar des autres citoyens, tout en leur accordant des privilèges particuliers liés à leur culte (la circoncision, le respect des fêtes, la possession d'un clergé reconnu, etc.). L'autre, défendue par son fils, Honorius, en 409, tient que les Juifs ne peuvent jouir pleinement de la protection des lois car «tout ce qui diffère de la foi chrétienne est contraire à la loi chrétienne $»^{11}$. Il ne faudrait toutefois pas se méprendre. En dépit de cette affirmation solennelle, les Juifs ont plein pouvoir sur l'exercice de leurs pratiques et l'organisation de la vie quotidienne de leurs coreligionnaires. Le droit romain tardif reconnaît l'entière juridiction des patriarches et des prélats, qui sont en outre dispensés des charges fiscales. Il s'agit de maintenir les privilèges antérieurs au passage à la chrétienté, qui affirmaient que les peuples avaient le droit de s'administrer eux-mêmes ${ }^{12}$. $\mathrm{Si}$ les Juifs disposaient d'une autonomie en matière religieuse que rien ne pouvait contrecarrer, la question de l'application du droit civil et familial, pan entier de la halakhah, mais dépassant les cadres de l'acception du religieux dans l'Empire, devenait cependant insoluble. Cela peut expliquer le maintien des tribunaux rabbiniques, devant lesquels les Juifs pouvaient porter leurs litiges civils. Les tribunaux rabbiniques bénéficièrent, comme les synagogues, d'une reconnaissance officielle, tandis que les décisions pénales prises entre Juifs continuaient à détenir force légale et devaient être exécutées par les magistrats impériaux. Protégés dans leur vie quotidienne, les Juifs ne pouvaient être contraints à

10. Amnon Linder, The Jews in Roman Legislation, Detroit - Jerusalem, Wayne State University Press- The Israel Academy of Sciences and Humanities, 1987, p. 33-50.

11. Amnon Linder, The Jews in Roman Legislation, op. cit., p. 64-65.

12. Ralph W. Mathisen, "The Citizenship and legal status of Jews in Roman law during late antiquity (ca300-540)", in John Tolan, et al. (éds.), Jews in Early Christian Law: Byzantium and the Latin West, 6th-11th Centuries. Turnhout, Brepols, 2014, p. 36-53, 40. 
profaner ni le chabbat ni leurs jours de fêtes. On observe ici la mise en place juridique d'un principe théologique : tout en protégeant le statut des Juifs dans la chrétienté, il les assujettissait à une position d'étrangeté permanente ${ }^{13}$. Ce principe garantit la liberté des Juifs et l'interdiction de les contraindre sous quelque forme que ce soit. Il a été énoncé très tôt, puis formulé explicitement par Grégoire ${ }^{\text {er }}$, dans une lettre adressée à l'évêque de Naples en 598. La formule Sicut iudeis renvoie cependant ordinairement à la bulle papale de Calixte II, édictée en 1120, puis reconduite régulièrement : "Tout comme les Juifs ne sont pas libres de franchir les limites imposées par la loi dans leurs communautés (ou synagogues), ils ne doivent de même souffrir d'aucun préjudice pour tout ce qui leur est autorisé $»^{14}$. Le $14^{\mathrm{e}}$ canon du Concile de Mâcon (581/3) ne dit pas autre chose : "Suivant l'édit décrété par le roi Childebert, il est interdit aux Juifs de s'égayer dans les rues principales et les places de marché à partir du Cena Domini (jeudi saint) jusqu'au premier jour des Pâques en raison de leur mépris; de plus, ils doivent être respectueux de tous les prêtres et du clergé du Seigneur, et ils ne doivent pas s'asseoir devant les prêtres, sauf ordre le leur précisant. Celui qui osera faire ce genre de choses sera puni par les iudices de ces lieux selon son statut ${ }^{15}$ ».

Ce principe d'autonomie légale et juridique accordé à une population favorise, voire encourage l'exercice d'un pouvoir interne - bien que restant tributaire des contraintes liées aux lois impériales - que l'on attribuera plus tard à celui du kahal, organisme central de la communauté au sens légal du terme. La communauté $^{16}$ italienne fonctionnait peut-être sur le modèle édifié jadis par le Sanhédrin de Jérusalem : organisée par un conseil (gerousia) et dirigée par des Anciens (presbyteroi: archontes).

13. A. Linder, The Jews in the legal sources of the Early Middle Ages, op.cit., p. 434.

14. Robert Austryn Markus, Gregory the Great and his world, Cambridge, Cambridge University Press, 1997, p. 77. L'analyse approfondie de cette bulle a été menée par Solomon Grayzel, « The Papal Bull Sicut Judeis », Meir Ben Horin, Bernard Dov Weinryb, Solomon Zeitlin, Studies and Essays in Honor of Abraham Neuman, Philadelphie, Leiden, Brill, 1962, p. 243-280.

15. Gregory I. Halfond, The Archeology of Frankish Church Councils ad 511-768, Leiden, Brill, 2010, p. 139.

16. Sur le sens du terme «communauté » dans la diaspora de l'Antiquité, voir Louis Feldman, Jew \& Gentile in the Ancient World, Princeton, Princeton University Press, 1996, p. 63-65. 
Ces derniers auraient eu en charge la réunion d'un fonds destiné à pourvoir aux besoins particuliers de la communauté (édifices, hôpitaux, écoles, cimetières), ainsi qu'au paiement des impôts et redevances, notamment aux prêtres de Jérusalem et aux autorités romaines (le fiscus judaicus). Parmi les fonctions communautaires, on trouve ainsi mention d'une sorte d'administrateur des biens, d'un secrétaire (grammateus), d'un patron protecteur (prostades), ainsi que d'un «père » ou d'une «mère » de la communauté ; fonction que les sources ne permettent pas de définir, mais dont les chercheurs supposent qu'il s'agissait d'un titre honorifique lié à leur générosité ou à des actes de bienfaisance ${ }^{17}$. Le terme archisynagoge, très répandu dans le monde gréco-romain, se retrouve également chez les Juifs, tant en Palestine que dans les autres régions. Il désigne le notable le plus honoré, le roch haknesset qui, assisté d'un bedeau, est responsable de l'ordonnancement liturgique du culte : il préside les assemblées religieuses, répartit les lecteurs lors des offices, délivre les homélies publiques et s'occupe des objets de la synagogue. Peut-on en déduire qu'il s'agit là des éléments embryonnaires que l'on retrouvera sous des appellations différentes dans les modèles communautaires ultérieurs du monde juif?

En dépit des mentions éparses d'événements ou de dispositions concernant les Juifs d'Occident entre l'Antiquité tardive et la fin du premier millénaire, il n'est nulle mention de communautés juives. On trouve, certes, de nombreuses observations relatives aux juifs ici et là : ils apparaissent dans la littérature ecclésiastique et la prose conciliaire ; ils sont mentionnés dans les chroniques de Grégoire de Tours au $\mathrm{VI}^{\mathrm{e}}$ siècle ${ }^{18}$, dans nombre de pamphlets et de traités, comme ceux d'Agobard de Lyon et de Bède au début du IX $X^{e}$ siècle. Ils composent un corpus recensé à fin du XIX ${ }^{e}$ siècle

17. J-B Frey, Corpus inscriptionum iudaicarum. Recueil des inscriptions juives qui vont du III e siècle avant Jésus-Christ au vII esiècle de notre ère. Vol I. Europe, Cité du Vatican, Rome, Institut Pontifical d'archéologie chrétienne, 1936 p. XCVI. P our une analyse plus détaillée de ces diverses fonctions, voir Harry J. Leon, The Jews of Ancient Rome [Philadelphie, 1960] Peabody, Hendrickson Publishers, 1995, le chap. "Organization of the Roman Jewish Community ", et Silvia Cappelletti, The Jewish Community of Rome. From the Second Century BC to the Third Century CE, Leyde, Boston, Brill, 2006, p. 3-30.

18. Grégoire de Tours, Histoire des Francs, trad. Robert Latouche, récemment rééditée en un volume, Paris, Les Belles Lettres, 2005. 
par Julius Aronius ${ }^{19}$, d'une valeur inestimable pour appréhender la vie des Juifs du Haut Moyen Âge, qui ont permis à Bernhard Blumenkranz de poser les bases de ses maitres ouvrages ${ }^{20}$, et l'on dispose à présent des sommes d'Amnon Linder et Walter Pakter qui ont amplement étoffé ces sources en compilant méthodiquement les sources juridiques et canoniques ${ }^{21}$. Aucun document issu du milieu juif ne permet toutefois de rendre compte de la manière dont les Juifs ont constitué des communautés (au sens juridique) ni du parcours migratoire qui les a conduits dans ces lieux. Or, dans la reconstitution historiographique qui a été effectuée, ces deux phénomènes sont intrinsèquement liés.

\section{LE TRAVAIL DE L'HISTORIOGRAPHIE}

Historiens et chercheurs se sont moins penchés sur les origines du premier Achkenaz - de l'Europe du Nord - que sur celles du second - de l'Europe de l'Est. La raison en est simple : cela demeure une énigme historique! Heinrich Graetz [1817-1891] l'avait résolue simplement: selon lui, le peuplement juif de l'Europe du Nord ne pouvait découler que de la capture de femmes palestiniennes par les légionnaires germaniques qui avaient participé à la bataille de Jérusalem. À leur retour victorieux après la défaite et la destruction du Temple, les légionnaires les auraient rapportées en Germanie parmi le butin et les prises de guerre. Là, elles auraient élevé seules leur progéniture, des générations durant, les éduquant et leur transmettant le judaïsme. Les ancêtres des juifs achkénazes auraient donc été à l'origine issus du fruit de l'asservissement sexuel des femmes palestiniennes ${ }^{22}$. Depuis

19. Julius Aronius, Regesten zur Geschichte der Juden im Fränkischen und Deutschen Reiche bis zum jähre 1273, Berlin, 1892.

20. Bernhard Blumenkranz, Juifs et chrétiens dans le monde occidental, Paris - La Haye, Mouton, 1960 ; ainsi que Les auteurs chrétiens latins du Moyen Age sur les juifs et le judaïsme, Paris - La Haye, Mouton \& Co, 1963.

21. Amnon Linder, The Jews in the legal sources of the Early Middle Ages, op. cit ; Walter Pakter, Medieval Canon Laws and the Jews, Ebelsbach, Verlag Rolf Gremer, 1988.

22. Heinrich Graetz, Geschichte der Juden von den ältesten Zeiten bis auf die Gegenwart, 11 vols. 1853-75, vol. 51 (1860), p. 188-189; V2 (1871), p. 166-169, Leipzig, Leiner, 2 1900, Berlin, Arani, 1998. Trad. anglaise, History of the Jews, Philadelphie, 1956, vol. 3, p. 40-41. 
Graetz, l'historiographie traditionnelle admet généralement que l'itinéraire migratoire qui a abouti à ces peuplements juifs provient de deux directions simultanées. L'une serait partie de l'Italie vers l'Allemagne et la France du Nord, l'autre, serait passée d'Espagne vers la Provence, puis de la Provence à la France du Nord et à l'Allemagne. Simon Schwarzfuchs et Avraham Grossman diffèrent sur l'importance à accorder à la migration espagnole dans la formation d'Achkenaz. S. Schwarzfuchs minimise l'impact de la voie hispanique ${ }^{23}$, A. Grossman, met à l'inverse l'accent sur le facteur des persécutions visigotiques dans la création des établissements juifs en Provence à la fin du $\mathrm{VII}^{\mathrm{e}}$ siècle, et dans le Languedoc après la conquête musulmane en $711^{24}$. Les travaux plus récents semblent, pour leur part, avoir renoncé à tenter de résoudre la question insoluble des origines de ce peuplement juif. Les chercheurs contemporains s'orientent plus généralement vers l'élaboration d'hypothèses concernant la réapparition des Juifs dans l'espace européen, suivie d'un surprenant accroissement démographique au cours des $\mathrm{IX}^{\mathrm{e}}$ et $\mathrm{X}^{\mathrm{e}}$ siècles. Asher Frishman propose, dans la récente synthèse historique qu'il présente du premier Achkenaz, de voir l'une des sources essentielles de la multiplication des Juifs en Europe dans les conversions des servantes travaillant à leur service ${ }^{25}$. Pourtant, rien ne permet d'affirmer que les Juifs qui revendiquent, au XIII ${ }^{\mathrm{e}}$ siècle, un lignage remontant jusqu'à la Judée ou à la Palestine n'en soient véritablement des descendants directs. Car s'il est certain que des établissements juifs ont existé en Gaule ${ }^{26}$ et en Germanie à l'époque romaine, il est fort probable que ceux qui furent à

23. Simon Schwarzfuchs, «L'opposition Tsarfat-Provence : la formation du judaïsme du nord de la France », Gérard Nahon (éd.), Hommages à Georges Vajda, Paris, Vrin, 1980, p. 135-150.

24. Avraham Grossman, «Immigration and Settlements in Germany in the Tenth-Eleventh Centuries », Avigdor Shinan (éd.), Immigration and Settlements in Jewish and general History (Heb.), Jerusalem, Zalman Shazar, 1982, p. 109-128; Id.«Communication among Jewish Centers during the Tenth to the Twelfth Centuries », Sophia Menache (éd), Communication in the Jewish Diaspora. The Pre-Modern world, Leyde, Brill, 1996, p. 107-125.

25. Asher Frishman, The Early Ashkenazi Jews. Since their Settlement in NorthWest Europe to the First Crusade ( Hébr.), Tel Aviv, Am Oved, 2008, p. 100.

26. Samuel Krauss a montré que la Gaule n'était pas inconnue des docteurs du Talmud, voir son «Antiquités gauloises dans le Talmud», Revue des études juives, 25, 1892, p. 14-29. 
l'origine de la formation des communautés et qui les peuplèrent plus tard, parvinrent en Europe du Nord via des trajectoires fort diverses, incluant tant Byzance que l'Orient.

Ne pouvant s'appuyer sur des sources inexistantes, c'est sur les aspects caractéristiques de la «tradition» développée en Europe du Nord que l'historiographie s'est concentrée. Or, si ses origines sont obscures, cette tradition achkénaze vaut qu'on tâche de comprendre comment elle s'est imposée au point de constituer l'un des marqueurs identitaires spécifiques du judaïsme de l'Europe du Nord $^{27}$. Furent ainsi convoqués les aspects typiques de la liturgie et de l'étude, mais aussi des pratiques sociales, et des structures institutionnelles. Les travaux portant sur la coutume achkénaze ont été principalement réalisés par quelques chercheurs : Moritz (Moshé) Güdeman, à la fin du XIX ${ }^{\mathrm{e}}$ siècle ${ }^{28}$, Irving Agus, dans les années cinquante du vingtième ${ }^{29}$, enfin plus récemment Ephraim Urbach $^{30}$, Joseph Tabory ${ }^{31}$, et plus spécifiquement encore Israël $\mathrm{Ta}^{-S h m a}{ }^{32}$ et Avraham Grossman ${ }^{33}$. Pour être un peu moins systématiques dans leurs approches, les travaux essentiels de Haym

27. Sylvie Anne Goldberg, «La 'Tradition' comme notion et comme principe dans le judaïsme rabbinique », in Madalina Vartejanu, Herméneutique et bricolage. Territoire et frontières de la Tradition dans le judaïsme, Berne, Peter Lang, 2008, p. $97-112$

28. Moritz (Moshé) Güdeman, [Geschichte des Erziehungswesen und der Cultur der abendländischen Juden während des Mittelalters, Vienne, 1880-1888], trad. héb. : Ha- torah vé-ha-hayyim bé-aratsot ha-maarav bé-yémé ha-benayim (la Torah et la vie dans les pays occidentaux au Moyen Âge), [Varsovie 1897] Jérusalem, 1962.

29. Irving Agus, The Heroic Age of Franco-Germany, New York, Yeshiva University Press, 1969 ; "The Oral Traditions of Pre-Crusades Ashkenazic Jewry », Studies and Essays in Honor of A. A. Neuman, op. cit.

30. Ephraim Elimélech Urbach, The Tosaphot: Their History, Writings and Methods (héb.), Jerusalem [1954], Bialik Institute, 2003.

31. Joseph Tabory and Méir Raffeld, Siddur (Ashkenazi - Hanau) 1628 (héb.), Ramat-Gan, Université Bar Ilan, 1984.

32. Israël M. Ta-Shma, Early Franco-German Ritual And Custom, (héb.), Jérusalem, The Magnes Press, The Hebrew University, 1992 ; Ritual, Custom and Reality in Franco-Germany, 1000 - 1350, (héb.), Jerusalem, Magnes Press, [1996] 2000 ; The Early Ashkenazic Prayer. Literary and Historical Aspects, (héb.), Jerusalem, Magnes Press, 2003.

33. Abraham Grossman, The Early Sages of Ashkenaz. Their Lives and Works (900-1096), (héb.) Jerusalem, Magnes Press [1981] 1988; The Early Sages of France. Their Lives, Leadership and Works (hébr.) Jerusalem, Magnes Press, 2001 ; Pious and Rebellious. Jewish Women in Medieval Europe [héb. 2001], Hanovre-Londres, Brandeis University Press, 2004. 
Soloveitchik ${ }^{34}$, Israël Yuval ${ }^{35}$, Ivan Marcus ${ }^{36}$, Talya Fishman ${ }^{37}$, Ephraim Karnafogel ${ }^{38}$, et probablement encore de bien d'autres ${ }^{39}$, qu'il serait impossible de recenser ici, traitent néanmoins également, d'une manière ou d'une autre, de ces questions de transmission et/ou de création de coutumes particulières. Un consensus se dégage ostensiblement de l'ensemble de ces travaux concernant l'une des caractéristiques, peut-être la plus avérée, de cet Achkenaz: sa singulière propension à l'accumulation et à la sacralisation de ses propres coutumes, leur faisant acquérir force de $\operatorname{loi}^{40}$. Mais, comment définir le contenu de ces coutumes particulières? Ce sont essentiellement des «manières de faire », qu'elles soient rattachées à l'étude et à l'enseignement, à la prière, ou encore aux formes de la structure communautaire et de la hiérarchie sociale. Ces coutumes déterminent ainsi l'agencement de la vie quotidienne mais aussi, surtout, les relations nouées avec les gouvernants extérieurs ${ }^{41}$. Et c'est justement ce dernier élément qui nous incite à revenir vers l'institution communautaire, sa constitution et son organisation. Jusque récemment, un consensus établissait en évidence si ce n'est en dogme que le modèle social et culturel palestinien d'Erets Yisrael avait été reconstitué et développé en Europe du Nord. Pour Yitzhak Baer - comme pour

34. Haym Soloveitchik, Principles and Pressures: Jewish Trade in Gentile Wine in the Middles Ages (héb.), Tel Aviv, Am Oved, 2003. ID ; « Religious Law and Change: The Medieval Ashkenazic example», American Jewish Studies Review, 12, 2, automne 1987, p. 205-221.

35. Isräl Jacob Yuval, Two Nations in Your Womb: Perceptions of Jews and Christians in Late Antiquity and the Middle Ages, [héb. 2000]Los Angeles \& Berkeley, University of California Press, 2006.

36. Ivan Marcus, Piety and Society. The Jewish Pietists of Medieval Germany, Leyde, Brill, 1981; Ritual of Childhood. Jewish Acculturation in Medieval Europe, New Haven-Londres, Yale University Press, 1996.

37. Talya Fishman, «Rhineland Pietist Approaches to Prayer and the Textualization of Rabbinic Culture in Medieval Northern Europe », Jewish Studies Quarterly, vol. 11 (2004), p. 313-331 ; « Rhineland Pietists' Sacralization of Oral Torah », Jewish Quarterly Review, 96, 1, 2006, p. 9-16.

38. Ephraim Karnafogel, Jewish Education and Society in the High Middle Ages. Wayne State University Press Detroit, 1992.

39. Dont le volume dirigé par Moshé Idel et Mortimer Ostow, Jewish Mystical Leadership in the 13 th Century, Lanham et al., Jason Aronson Book, [1998] 2005.

40. Israël M. Ta Shma, Early Franco-German Ritual And Custom, op. cit., introduction, p. 15-16.

41. S. A. Goldberg, «Common Law and Jewish Law. The Diasporic Principle of Dina de-Malkuta Dina », Behemoth. A Journal on Civilization, 2008, 2, p. 39-53. 
ben Zion Dinur d'ailleurs - la qehillah incarnait le pilier qui avait permis à la vie juive de se maintenir en exil. Baer voyait une continuité de cette institution collective à partir de la période du Second Temple, avec l'établissement d'un centre, rayonnant par-delà les frontières, lui permettant de se perpétuer en toute indépendance, et de dépasser le traumatisme de la destruction. Toutefois, écrit-il: "L'aspect démocratique de la qehillah a été conservé en Erets Yisrael et renouvelé durant la période des géonim, et c'est de là que proviennent les bases figurant l'organisation de la qehillah dans l'Europe du Moyen Âge [...] qui se sont maintenues plus de 1300 ans $»^{42}$.

Mais de quoi s'agit-il, au juste, lorsqu'on parle de qehillah? Tout d'abord de l'organisation, même rudimentaire, de la vie juive. L'exercice du judaïsme, puisqu'il implique des obligations collectives, rend nécessaire la mise en œuvre d'une sorte de service public efficace. Il en va ainsi, par exemple, de l'organisation des rituels quotidiens: les offices devant être célébrés en mynian, le minimum de dix personnes (mâles) formant le quorum, l'édification ou la transformation d'un espace quelconque en lieu de prière est indispensable. À ce bâtiment, dans lequel pouvait se trouver une salle de réunion ou d'enseignement devait probablement s'ajouter le bain rituel, le miqvèh, situé dans un bâtiment attenant, maison privée ou communautaire. Puis, peu à peu, vient s'ajouter la propriété d'un cimetière particulier, qui finit par servir de référence d'appartenance à une communauté donnée ${ }^{43}$. Enfin, en dépit du fait que rien ne permet vraiment d'éclairer la manière dont les juifs de l'Europe du Nord ont conçu leurs formes d'administration et de gestion de l'autorité, force est de constater que le tribunal rabbinique, veillant à l'application de la loi juive sur ses administrés, est l'une des institutions les plus anciennes du monde achkénaze. Le Qahal, personne morale de la qehillah, est

42. Yitshak Baer, «Ha-yesodot vé-ha-tehalot chel irgoun ha-qehillah ha-yehoudit be-yemei ha-benayim: The Origins of the Organisation of the Jewish Community of the Middle Ages "Studies in the History of the Jewish People, (Hébr. 2 vols), Jérusalem, The Historical Society of Israel, 1985, II, p. 60-100 ; p. 22-81 (Zion, 15, 1950, p. 1-41)

43. L'émergence des cimetières juifs européens correspond à celle des communautés organisées. Il n'en existe aucune trace remontant à une date antérieure au XI ${ }^{\mathrm{e}}$ siècle. Voir S. A. Goldberg, Les Deux rives du Yabbok, Paris, Cerf, 1989, p. 37-41. 
ainsi responsable de l'existence et du maintien de la synagogue (beit ha-knesset), du bain rituel et du cimetière, pour l'entretien desquels il est nécessaire de prélever des impôts. De sorte que la communauté, en se développant, va également s'attribuer un certain nombre de prérogatives, qui restent spécifiques aux contrées d'Achkenaz, qui s'exprimeront dans la formulation d'édits particuliers, les taqqanot, destinés à réguler la bonne marche de la vie de leurs administrés. Parmi ceux-ci, on en trouve notamment trois qui visent à prévenir les conflits et les rivalités internes : 1) l'édit du ban de l'implantation, herem ha-yichouv, qui restreint l'établissement de nouveaux venus en le soumettant à l'accord des habitants autochtones; 2) l'édit qui permet la suspension de la prière publique (ikouv ha-tefilah) à la synagogue, lorsqu'un individu souhaite se faire rendre justice et jusqu'à ce que sa plainte soit traitée ; 3) l'édit de monopole ou de ma'arifa, qui prescrit l'exclusivité des relations commerciales (ma'aroufiah) entretenues notoirement entre un juif et son client (non-juif) particulier, interdisant de la sorte à aucun autre de ses coreligionnaires de s'immiscer en concurrence dans cette association ${ }^{44}$.

Il n'empêche. Dans l'introduction d'un symposium sur les Juifs d'Europe au Moyen Âge, Alfred Haverkamp souligne qu'il n'existe aucune mention de «communautés » au sens juridique avant le $\mathrm{x}^{\mathrm{e}}$ siècle, et qu'il en va de même pour les chrétiens qui n'ont pas plus de système communal avant cette période ${ }^{45}$. L'apparition du Qahal participe, en fait, du phénomène d'urbanisation de la société médiévale. Regroupées sous l'appellation de Choum, les communautés de Spire, Worms et Mayence, dont les initiales hébraïques de leurs villes (Chpiyer, Wermeza, Magenza) forment le nom, élaborent cette première forme de gouvernement

44. Irving Agus, The Heroic Age of Franco-Germany, New York, Yeshiva University Press, 1969, chap. « Monopoly »; A. Grossman, « The Jewish Community in Ashkenaz in the 10-11 $1^{\text {th }}$ Centuries », A. Grossman and Yosef Kaplan (éds), Klal Yisrael. Jewish Self-Rule Through the Ages. Vol. 2: The Middle Ages and Early Modern Period (Hébr.), Jérusalem, Zalman Shazar, 2004, p.57-74 ; Simon Schwarzfuchs, Kahal. La communauté juive de l'Europe médiévale, Paris, Maisonneuve et Larose, 1986.

45. Alfred Haverkamp, «The Jews of Europe in the Middle Ages: By way of Introduction», Christoph Cluse (ed.), The Jews of Europe in the Middle Ages (Tenth to Fifteen Centuries). Proceedings of the International Symposium held at Speyer, 20-25 October 2002, Cultural Encounters in Late Antiquity and the Middle Ages, Turnhout, Brepols, 2004, p. 2. 
régional. S'établissant au cœur des cités, édifiant des quartiers ou des rues parfois protégés par des enceintes ${ }^{46}$, dont les abords des cathédrales ou des châteaux découvrent les vestiges, les juifs instituèrent des taqqanot, autrement dit des règlements généraux, dont relevaient également ceux qui vivaient isolés dans les villages de la campagne environnante. En tête de ces règlements, on trouve dorénavant la mention systématique de qehilla qeducha: Sainte communauté. Cette formulation semble banale. On pourrait même ne pas s'y arrêter tant elle relève de la phraséologie typiquement médiévale, juive comme chrétienne. Elle n'en relève pas moins pour autant d'un bagage théologique qui, pour sa part, n'est rien moins qu'anodin et mérite qu'on lui prête attention.

\section{Une COMMUNAUTÉ DE DESTIN}

Les historiens juifs ont souvent été frappés par l'accent porté sur l'idée du collectif, le klal Yisrael, dans les présentations et les tournures des énoncés des taqqanot achkénazes médiévales. Ainsi l'expression kol yisrael arevim zé le-zé qui signifie que chacun est responsable de tous, renvoie-t-elle, naturellement aux conceptions théologiques qui ont été évoquées au tout début de cet article. Il serait aisé, d'ailleurs, d'identifier une continuité entre la Sainte communauté de Jérusalem que j'ai brièvement mentionnée plus haut et les Saintes communautés de Choum. Or, rien, hormis l'expression elle-même, ne permet de la suivre dans l'histoire. C'est donc ailleurs qu'il faut chercher le sens de ce klal - et c'est sur ce dernier point que je vais conclure. Comme l'a pointé judicieusement Avraham Grossman, la littérature scientifique juive a généralement décrit ces communautés comme réalisant parfaitement l'idéal d'une «communauté sainte» par l'intermédiaire de son emprise sur la collectivité, mettant ainsi en œuvre l'idée de « bien commun » plutôt que celle du bien de l'individu. Pour dire autrement ce que recouvre cet idéal de bien collectif, il faut préciser que la communauté est

46. Charte de l'évêque Rüdiger édictée pour les Juifs de Spire en 1084, elle sera suivie, peu après, par la promulgation de semblables privilèges à la communauté de Worms. Louis Wirth, Le Ghetto, Grenoble, Presses universitaires de Grenoble,1980, p. 43-44. 
mandatée pour agir au nom de la rédemption collective d'Isrä̈l ${ }^{47}$. C'est ce que résume Simon Schwarzfuchs: «l'idéal de vie du Juif médiéval est résolument communautaire: que des pressions extérieures l'aient contraint au séjour dans la ville ou la bourgade ou qu'il l'ait élu de son plein gré ne change rien à l'affaire [...] la communauté sera donc le cadre de vie que recherchera de préférence quiconque adhère à cet idéal $»^{48}$. C'est encore dans cette perspective que S. Schwarzfuchs cite le Sefer hasidim $[\$ 116,74]$ : «Si les Juifs n'étaient pas garants l'un pour l'autre (kol yisrael arevim zé $l e ́-z e ́)$, nul ne ferait de reproches à son prochain et ne s'efforcerait de supprimer les malfaisants. Cette solidarité leur fait un devoir d'établir les barrières et les haies qui les empêcheront d'inciter au péché ${ }^{49}$. C'est pourtant dans l'insistance portée par les auteurs des règlements communautaires sur l'aspect sacré de leurs décisions entre les $\mathrm{XI}^{\mathrm{e}}$ et le XIII ${ }^{\mathrm{e}}$ siècle que l'on peut lire, entre les lignes, les difficultés rencontrées par les autorités rabbiniques afin d'imposer leur emprise sur leurs coreligionnaires. De sorte qu'en transférant les règles civiles ou pénales (traitant du commerce, de la concurrence ou du mariage et de l'héritage) sur le plan de la rédemption collective de « l'ensemble d'Israël » ces derniers usaient à l'égard des leurs des mêmes politiques eschatologiques que les chrétiens avaient mises en œuvre à l'encontre des Juifs : ils les plaçaient ainsi dans une sorte de salle d'attente destinée à ouvrir sur la fin des temps.

Les relations ambiguës nouées entre Juifs et chrétiens ont provoqué des effets miroirs qui se sont répercutés dans les évolutions respectives des deux religions en présence et que l'on commence à appréhender de manière plus aiguë. Ainsi Israël Yuval a su démontrer avec fracas et brio les allers-retours développés entre Juifs et Chrétiens dans la rhétorique de la haine ${ }^{50}$. Robert Chazan a, quant à lui, exposé le rôle que les exégèses autour de la prophétie, et notamment celle d'Isaïe sur le Serviteur de Dieu, avaient joué

47. Avraham Grossman, «ha qehillah ha-yehoudit be-Ashkenaz be-yemei ha-beinayim: bein partikularizm le - 'klal Yisrael' (La communauté juive médiévale en Ashkénaz: entre particularisme et l'ensemble d'Israël) », Shmuel Noah Eisenstadt and Moshe L. Lissak, Ha-tsionout ve-ha-hazarah le-historiah (Le sionisme et le retour vers l'histoire), Jérusalem, Yad Ben Tsevi, 1999, p. 166-179.

48. Simon Schwarzfuchs, Kahal. La communauté juive de l'Europe médiévale, Paris, Maisonneuve \& Larose, 1986, p. 133.

49. Ibid, p. 137.

50. Israël Yuval, Deux peuples en ton sein, Paris, Albin Michel, 2012. 
dans la définition de la catégorie «juif» durant le Moyen Âge ${ }^{51}$. Pour ma part, je me contenterai de revenir à l'analyse du concept tiré de la lecture biblique du « reste d'Israël », à partir des échanges développés entre Chrétiens et Juifs. La transposition effectuée par Eusèbe, et reprise comme un acquis par les Pères de l'Église, avait permis de faire passer les rescapés d'Israël dans les rangs des élus qui avaient su entendre et transmettre le message divin. Pour les Juifs, par contre, les ché'érit, le « reste », faisait, on l'a dit, partie d'un «tout», l'ensemble du peuple qui n'avait pas disparu. La notion de péché, toutefois, pose une condition à la réalisation de cet idéal du collectif car, comme nous le rappelle Rachi : "che'ar yachouv - ha-ché'érit che ba-hen yachouvou el ha-qadoch baroukh hou : le reste d'entre eux reviendra à Dieu Béni Soit-Il ». La question de la rédemption collective se pose ici en effet avec acuité, car ceux qui «reviendront» à Dieu ne seront qu'un ché'érit, un «reste». On saisit mieux, dès lors, l'importance que la pensée juive accorde à la sainteté (qedouchah) qu'il est nécessaire de faire appliquer à l'ensemble de la communauté (qehillah). Et l'on saisit également l'importance que la pensée chrétienne accorde à la conservation voire à la fossilisation des vestiges de l'ancien Israël en vue de leur conversion ultime. Quant à la formation médiévale de la «nation juive », qui, faut-il le rappeler, ne répond en rien aux critères actuels de la définition qui associe un peuple à un État, elle résulte, comme on le sait, des mesures de discrimination qui ont conduit graduellement à distinguer un groupe humain qui, des siècles durant, n'était aucunement distinguable de ceux qui les environnaient.

Accordant au discours théologique la propriété d'être intemporel et de transcender le temps et les contextes historiques en modifiant son expression tout en propageant les mêmes antiennes, l'époque moderne a vu se développer le dessein d'en finir avec le pouvoir collectif qui allait de pair avec l'autonomie de la Communauté juive. Ne pourrait-on pas se demander cependant si ce n'était pas pour mieux faire retour dans la stigmatisation, non plus d'une autorité légale, mais du simple pouvoir accordé à une affiliation - même si celle-ci relève parfois du seul ressort de l'imaginaire ?

sag@ehess.fr

51. Robert Chazan,, Fashioning Jewish Identity in Medieval Western Christendom, Cambridge, Cambridge University Press, 2004. 